\title{
Editorial
}

\section{The Journal's Mission and Standards of Presentation}

The mission of Communications in Mathematical Physics is to offer a high forum for works which are motivated by the vision and the challenges of modern physics and which at the same time meet the highest mathematical standards.

The above is a broad calling, as it encompasses different subfields, which in practice have varying foundations, different subcommunities of practitioners, and which may be at different stages of progress towards mathematical maturity.

As a primary instruction, we call upon the authors to keep both the above stated goals and the broad audience in mind, and we offer the following suggestions to this end.

- State the results in a broadly accessible way.

- Bear in mind that the case for publication in CMP is enhanced by a clear indication of the work's relation to physics issues, or to mathematics which have sprung from such a relation.

- Ensure that the status of the results presented in the work is unambiguously understood: whether they are established rigorously, i.e. proven within a mathematically precise framework, or according to other standards, for example granting the validity of some particular physical framework.

- As possible, formulate clear definitions and state theorems, as facilitated by the formalism used. Conjectures, clearly stated as such, also have their place.

Finally, it is important to put the work into context, stating for a broad audience both the background and the essential advances being made.

While none of this is new, and the best papers in CMP have always exemplified these standards, the breadth of our readership as well as of our contributors makes it appropriate to reemphasize the importance of these requirements. They can be met in different ways; one possibility is to include an introductory section aimed at a broader readership than the body of the paper, where one may assume a somewhat higher level of familiarity with the subject.

Both authors and referees are asked to consider these requirements. In some areas this may be viewed as an addition of another hurdle to an already demanding refereeing process, but we see this as an important part of the growth of mathematics and physics. We hope that the authors, referees, and readers will agree that the goals, which include a strong field and a vital mathematical physics community, are well worth this effort. 